\title{
Langmuir
}

pubs.acs.org/Langmuir

(C) 2010 American Chemical Society

\section{Langmuir and Gibbs Magnetite NP Layers at the Air/Water Interface}

\author{
Cristina Stefaniu, ${ }^{* \dagger}{ }^{\dagger}$ Munish Chanana ${ }^{\dagger}$ Dayang Wang, ${ }^{\dagger}$ Dmitri V. Novikov, ${ }^{*}$ \\ Gerald Brezesinski, ${ }^{* \dagger}$ and Helmuth Möhwald ${ }^{\dagger}$
}

\begin{abstract}
${ }^{\dagger}$ Max Planck Institute of Colloids and Interfaces, Science Park Potsdam-Golm, Am Muehlenberg 1, D-14476 Potsdam, Germany, and ${ }^{\star} H A S Y L A B$ at DESY, Notkestr. 85, 22607 Hamburg, Germany
\end{abstract}

Received October 1, 2010. Revised Manuscript Received November 16, 2010

\begin{abstract}
The interfacial properties of $\mathrm{Fe}_{3} \mathrm{O}_{4} @ \mathrm{MEO}_{2} \mathrm{MA}_{90}$-co-OEGMA ${ }_{10} \mathrm{NPs}$, recently developed and described as promising nanotools for biomedical applications, have been investigated at the air/water interface. These $\mathrm{Fe}_{3} \mathrm{O}_{4} \mathrm{NPs}$, capped with catechol-terminated random copolymer brushes of 2-(2-methoxyethoxy) ethyl methacrylate $\left(\mathrm{MEO}_{2} \mathrm{MA}\right)$ and oligo(ethylene glycol) methacrylate (OEGMA), with molar fractions of $90 \%$ and $10 \%$, respectively, proved to be surface active. Surface tension measurements of aqueous dispersions of the NPs showed that the adsorption of the NPs at the air/water interface is time- and concentration-dependent. These NPs do not behave as classical amphiphiles. Once adsorbed at the air/water interface, they do not exchange with NPs in bulk, but they are trapped at the interface. This means that all NPs from the bulk adsorb to the interface until reaching maximum coverage of the interface, which corresponds to values between $6 \times 10^{-4}$ and $8 \times 10^{-4} \mathrm{mg} / \mathrm{cm}^{2}$ and a critical equilibrium surface tension of $\sim 47 \mathrm{mN} / \mathrm{m}$. Moreover, Langmuir layers of $\mathrm{Fe}_{3} \mathrm{O}_{4} @ \mathrm{MEO}_{2} \mathrm{MA}_{90}$-co-OEGMA ${ }_{10}$ NPs have been investigated by measuring surface pressure-area compression-expansion isotherms and in situ X-ray fluorescence spectra. The compression-expansion isotherms showed a plateau region above a critical surface pressure of $\sim 25 \mathrm{mN} / \mathrm{m}$ and a pronounced hysteresis. By using a special one-barrier Langmuir trough equipped with two surface pressure microbalances, we have shown that the NPs are squeezed out from the interface into the aqueous subphase, and they readsorb on the other side of the barrier. The results have been supported by TEM as well as AFM experiments of transferred Langmuir-Schaefer films on solid supports. This study shows the ability of $\mathrm{Fe}_{3} \mathrm{O}_{4} @ \mathrm{MEO}_{2} \mathrm{MA}_{90}$-co-OEGMA 10 NPs to transfer from hydrophilic media (an aqueous solution) to the hydrophobic/hydrophilic interface (air/water interface) and back to the hydrophilic media. This behavior is very promising, opening studies of their ability to cross biological membranes.
\end{abstract}

\section{Introduction}

In the past decade, iron oxide nanoparticles proved to be very attractive for medical purposes due to their unique features, such as their large surface/volume ratio, which allows them to bind, adsorb, or carry compounds such as drugs, proteins, enzymes, or antibodies, ${ }^{1}$ which can be then directed to a special tissue or organ using an external magnetic field or can be heated in alternating magnetic fields for use in hyperthermia, ${ }^{2}$ and so on. Therefore, in the biomedical field, ${ }^{2}$ the iron oxide nanoparticles are very useful as magnetic resonance imaging contrast enhancers (clinical diagnosis), ${ }^{3,4}$ as inflammation response or anticancer agents, ${ }^{5}$ for labeling and cell separation, ${ }^{6}$ as drug delivery systems, ${ }^{7-9}$ and so forth.

The magnetic nanoparticles have attracted great attention in materials science research for the development of magnetic-data

(1) Jong, W. H. D.; Borm, P. J. Int. J. Nanomed. 2008, 3(2), 133-149.

(2) Gupta, A. K.; Gupta, M. Synthesis and surface engineering of iron oxide nanoparticles for biomedical applications. Biomaterials 2005, 26, 3995-4021.

(3) Corot, C.; Robert, P.; Idée, J.-M.; Port, M. Recent advances in iron oxide nanocrystal technology for medical imaging. Adv. Drug Delivery Rev. 2006, 58, 1471-1504.

(4) Kircher, M. F.; Allport, J. R.; Graves, E. E.; Love, V.; Weissleder, R. Cancer Res. 2003, 63, 6838-6846.

(5) J. Meng, J. F.; Galiana, G.; Branca, R. T.; Clasen, P. L.; Ma, S.; Zhou, J.; Leuschner, C.; Kumar, C. S. S. R.; Hormes, J.; Otiti, T.; Beye, A. C.; Harmer, M. P.; Kiely, C. J.; Warren, W.; Haataja, M. P.; Soboyejo, W. O. LHRH-functionalized superparamagnetic iron oxide nanoparticles for breast cancer targeting and contrast enhancement in MRI. Mat. Sci. Eng., C-Bio S 2009, 29(4), 1467-1479.

(6) Josephson, L.; Tung, C.-H.; Moore, A.; Weissleder, R. High-efficiency intracellular magnetic labeling with novel superparamagnetic-tat peptide conjugates. Bioconjugate Chem. 1999, 10(2), 186-191.

(7) Butoescu, N.; Seemayer, C. A.; Palmer, G.; Guerne, P. A.; Jordan, O Arthritis Res. Ther. 2009, 11, 3 .

(8) Gupta, A. K.; Curtis, A. S. G. Biomaterials 2004, 25, 3029-3040.

(9) Gupta, A. K.; Gupta, M. Biomaterials 2005, 26, 1565-1573. recording media and nanocomposite permanent magnets, ${ }^{10}$ and from catalytic chemistry as well as from colloid chemistry, ${ }^{11}$ where the ferrofluids represent a topic of high interest.

In the present paper, we report on the original interfacial behavior of $\mathrm{Fe}_{3} \mathrm{O}_{4}$ NPs. These NPs grafted with the thermosensitive and biocompatible $\mathrm{MEO}_{2} \mathrm{MA} / \mathrm{OEGMA}$ copolymer proved to be very promising for biomedical use. ${ }^{12}$ The motivation of the study comes from the question concerning the ability of these $\mathrm{Fe}_{3} \mathrm{O}_{4}$ NPs to cross biological membranes. To do so, the NPs must have a hydrophilic character in order to be well-accommodated in the extracellular space; after the insertion into the cellular membrane they will need to be hydrophobic and, for release into the intracellular space, they will need to be able to again become hydrophilic. Taking all this into account and to simplify this hydrophilic/hydrophobic/hydrophilic system, we studied in the first step the properties of the NPs at the simplest hydrophilic/ hydrophobic interface, the water/air interface.

The scientific literature offers an important number of papers dedicated to the formation, the organization, and the stability of Langmuir films of iron oxide $\left(\mathrm{Fe}_{2} \mathrm{O}_{3}\right.$ or $\left.\mathrm{Fe}_{3} \mathrm{O}_{4}\right)$ NPs and their Langmuir-Blodgett transfer. For example, Fendler et al., ${ }^{13}$ as well as Kang et al., ${ }^{14}$ studied surfactant-stabilized $\mathrm{Fe}_{3} \mathrm{O}_{4}$ NPs at the air/water interface by both transmission electron microscopy

(10) Zeng, H.; Li, J.; Liu, J. P.; Wang, Z. L.; Sun, S. Nature 2002, 420, 395

(11) Amstad, E.; Gillich, T.; Bilecka, I.; Textor, M.; Reimhult, E. Ultrastable iron oxide nanoparticle colloidal suspensions using dispersants with catecholderived anchor groups. Nano Lett. 2009, 9(12), 4042-4048.

(12) Chanana, M.; Jahn, S.; Georgieva, R.; Lutz, J.-F.; Bäumler, H.; Wang, D. Chem. Mater. 2009, 21(9), 1906-1914.

(13) Meldrum, F. C.; Kotov, N. A.; Fendler, J. H. J. Phys. Chem. 1994, 98, 4506.

(14) Lee, D. K.; Kim, Y. H.; Kim, C. W.; Cha, H. G.; Kang, Y. S. J. Phys. Chem. B 2007, 111, 9288-9293. 


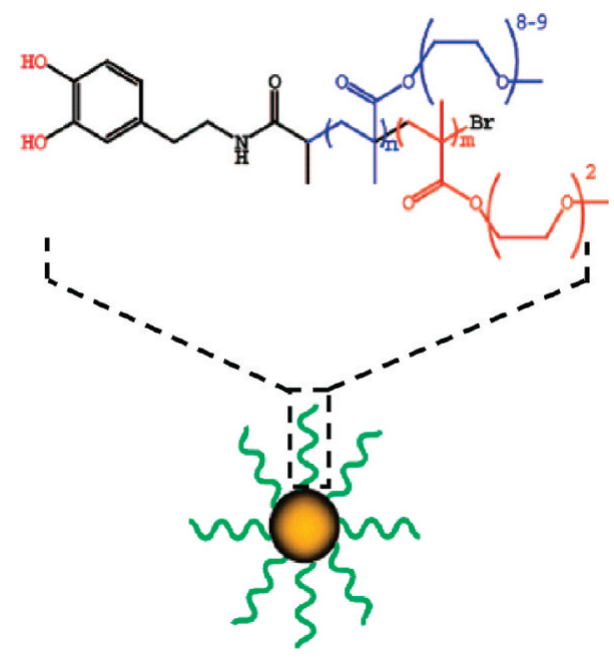

Figure 1. Schematic representation of the $\mathbf{F e}_{3} \mathbf{O}_{4} @ \mathbf{M E O}_{2} \mathbf{M A}_{\mathbf{9 0}^{-}}$ co-OEGMA 10 NPs.

(TEM) and Brewster angle microscopy (BAM). Paulus et al. used $\mathrm{X}$-ray reflectivity experiments for studying the adsorption of positively charged $\gamma-\mathrm{Fe}_{2} \mathrm{O}_{3}$ (maghemite) NPs from an aqueous colloidal suspension at the air/water interface, ${ }^{15}$ as well as at different monolayers. ${ }^{16}$ On the basis of pressure-molecular area $(\pi / A)$ isotherms and atomic force microscopy measurements, Kressler et al. ${ }^{17}$ reported on Langmuir monolayers and LangmuirBlodgett (LB) film morphologies formed by mixtures of amphiphilic block copolymers (poly(ethylene oxide)/poly(isobutylene)) and iron oxide $\left(\mathrm{Fe}_{2} \mathrm{O}_{3}\right)$ nanoparticles capped with oleic acid. Stroeve et al. ${ }^{18}$ studied the behavior of a Langmuir layer of poly(octadecene-co-maleic anhydride) on a subphase of a colloidal aqueous suspension (hydrosol) of magnetic $\mathrm{Fe}_{3} \mathrm{O}_{4}$ nanoparticles. The influence of the NPs on the polymer monolayer has been observed by the formation and growth of domains and was explained by electrostatic interactions of the anionic carboxylate groups of the copolymer with the cationic surface charges of the $\mathrm{Fe}_{3} \mathrm{O}_{4}$ nanoparticles. Later on, Stroeve et al. ${ }^{19}$ prepared ultrathin films of $\mathrm{CoFe}_{2} \mathrm{O}_{4}$ nanocrystallites at the air/water interface exhibiting a remarkable degree of long-range order.

The system studied in the present work is completely different from the iron oxide NPs already studied at the air/water interface due to the unique characteristics of the copolymer used. It consists of a monodisperse population of $\mathrm{Fe}_{3} \mathrm{O}_{4}$ cores with a diameter of $6.4 \mathrm{~nm}$, which are grafted with catechol-terminated copolymers of 2-(2-methoxyethoxy) ethyl methacrylate $\left(\mathrm{MEO}_{2} \mathrm{MA}\right)$ and oligo(ethylene glycol) methacrylate (OEGMA) ${ }^{12}$ (Figure 1). The NPs were named $\mathrm{Fe}_{3} \mathrm{O}_{4} @ \mathrm{MEO}_{2} \mathrm{MA}_{90}$-co-OEGMA 10,90 and 10 representing the molar fractions of $\mathrm{MEO}_{2} \mathrm{MA}$ and OEGMA, respectively. It is important to highlight the fact that these NPs are dual-dispersible. Due to the presence of oligo(ethylene glycol) side groups on the surfaces, the $\mathrm{Fe}_{3} \mathrm{O}_{4} @ \mathrm{MEO}_{2} \mathrm{MA}_{90}$-co-OEGMA 10 NPs can be dispersed in water, exhibiting a high colloidal stability against salt, and at the same time, they can be well-dispersed in organic solvents, like chloroform, ethanol, or toluene. This

(15) Paulus, M.; Degen, P.; Schmacke, S.; Maas, M.; Kahner, R.; Struth, B.; Tolan, M.; Rehage, H. Eur. Phys. J. Special Topics 2009, 167, 133-136.

(16) Degen, P.; Paulus, M.; Maas, M.; Kahner, R.; Schmacke, S.; Struth, B.; Tolan, M.; Rehage, H. Langmuir 2008, 24, 12958-12962.

(17) Li, H.; Sachsenhofer, R.; Binder, W. H.; Henze, T.; Thurn-Albrecht, T.; Busse, K.; Kressler, J. Langmuir 2009, 25(14), 8320-8329.

(18) Kang, Y. S.; Risbud, S.; Rabolt, J.; Stroeve, P. Langmuir 1996, 12, 43454349 .

(19) Lee, D. K.; Kim, Y. H.; Kang, Y. S.; Stroeve, P. J. Phys. Chem. B 2005, 109, 14939-14944. specific copolymer in water (no salt) is hydrophilic and becomes hydrophobic by increasing the temperature above $40{ }^{\circ} \mathrm{C}$.

The present work was focused on the study of the $\mathrm{Fe}_{3} \mathrm{O}_{4} @$ $\mathrm{MEO}_{2} \mathrm{MA}_{90}$-co-OEGMA 10 NPs at the air/water interface by measuring their dynamic adsorption and Langmuir compressionexpansion isotherms. The Langmuir films have been characterized in situ by X-ray fluorescence or after Langmuir-Schaefer transfer onto solid support by TEM and AFM. We obtained surprising but consistent results on the partitioning of the NPs between bulk and interface.

\section{Experimental Section}

Materials. The $\mathrm{Fe}_{3} \mathrm{O}_{4} @ \mathrm{MEO}_{2} \mathrm{MA}_{90}$-co-OEGMA 10 NPs (6.4 nm diameter of the metallic core; $4.8 \mathrm{~nm}$ polymer shell thickness and $M_{\mathrm{n}}=39000 \mathrm{~g} \cdot \mathrm{mol}^{-1}$ ) used in this study were synthesized and characterized as previously reported. ${ }^{12}$ The NPs are not charged, and they have a weight fraction of $29.5 \%$ of $\mathrm{Fe}_{3} \mathrm{O}_{4}$ (thermogravimetric analysis, TGA). Moreover, special attention has been paid to the purity of the NP system with respect to free polymer. The calculations made (see Supporting Information), using the critical interfacial concentrations of the NPs-copolymer system and of the copolymer alone at the interface, indicate that there is no free copolymer in the studied NP system. The NPs have been dispersed at different concentrations, either in Milli-Q Millipore water with a specific resistance of $18.2 \mathrm{M} \Omega \cdot \mathrm{cm}$ or in chloroform (HPLC grade).

Tensiometer Measurements. The dynamic surface tension measurements have been performed with a profile analysis tensiometer (PAT-1, Sinterface, Germany). The value of the surface tension was determined from the shape of the pendant drops. ${ }^{20}$ The volume of the drop was kept constant by an active control loop of this instrument. For all the experiments, aqueous drops of different concentrations of $\mathrm{Fe}_{3} \mathrm{O}_{4} \mathrm{NPs}$ were formed in air, and the dynamic surface tension was measured over several hours, in order to reach the equilibrium surface tension. All experiments have been performed at room temperature $(21 \pm 1){ }^{\circ} \mathrm{C}$.

Surface Pressure-Area Isotherms. The pressure-area isotherms of the $\mathrm{Fe}_{3} \mathrm{O}_{4} @ \mathrm{MEO}_{2} \mathrm{MA}_{90}$-co-OEGMA 10 NPs at the air/ water interface were measured with a Langmuir trough system equipped with one (or two) moving barriers. The setup included one (or two) surface pressure microbalances with filter paper Wilhelmy plates. The results were plotted as surface pressure $(\pi)$ versus the area of the trough (in $\mathrm{cm}^{2}$ ). The bare water surface was proven to be clean by compression before each measurement. The temperature of the Milli-Q Millipore water subphase was maintained at $20^{\circ} \mathrm{C}$ by using a circulating water bath.

Different amounts of chloroform solutions of the NPs $(3 \mathrm{mg} / \mathrm{mL})$ were uniformly spread on the subphase by using a microsyringe (Hamilton). The compression of the film, at a constant rate of $10.8 \mathrm{~cm}^{2} / \mathrm{min}$, was started $20 \mathrm{~min}$ after spreading to ensure the complete evaporation of the solvent and the uniform distribution of the NPs at the interface. The pressure/area $(\pi / A)$ isotherms were recorded during compression of the monolayer on the computer-interfaced Langmuir trough ( $\& \& \mathrm{~K}$, Potsdam, Germany). Each measurement was repeated at least 2 times to prove the reproducibility of the results. In order to avoid dust contamination of the interface and to ensure a constant humidity, the Langmuir trough was placed in a sealed box.

In order to prove that the NP monolayer does not slide underneath the barrier to the other compartment, two different kinds of barriers have been used: one barrier made of Teflon (polytetrafluoroethylene) and another one made of POM (polymethylene oxide). Due to the high hydrophobicity of the Teflon barrier (the water contact angle on Teflon is $101^{\circ}$ ), the water is repelled from

(20) Loglio, G.; Pandolfini, P.; Miller, R.; Makievski, A. V.; Ravera, F.; Ferrari, M.; Liggieri, L., Drop and bubble shape analysis as tool for dilational rheology studies of interfacial layers. In Novel Methods to Study Interfacial Lavers, Möbius, D., Miller, R., Eds.; Elsevier: Amsterdam, 2001; Vol. 11, p 439. 

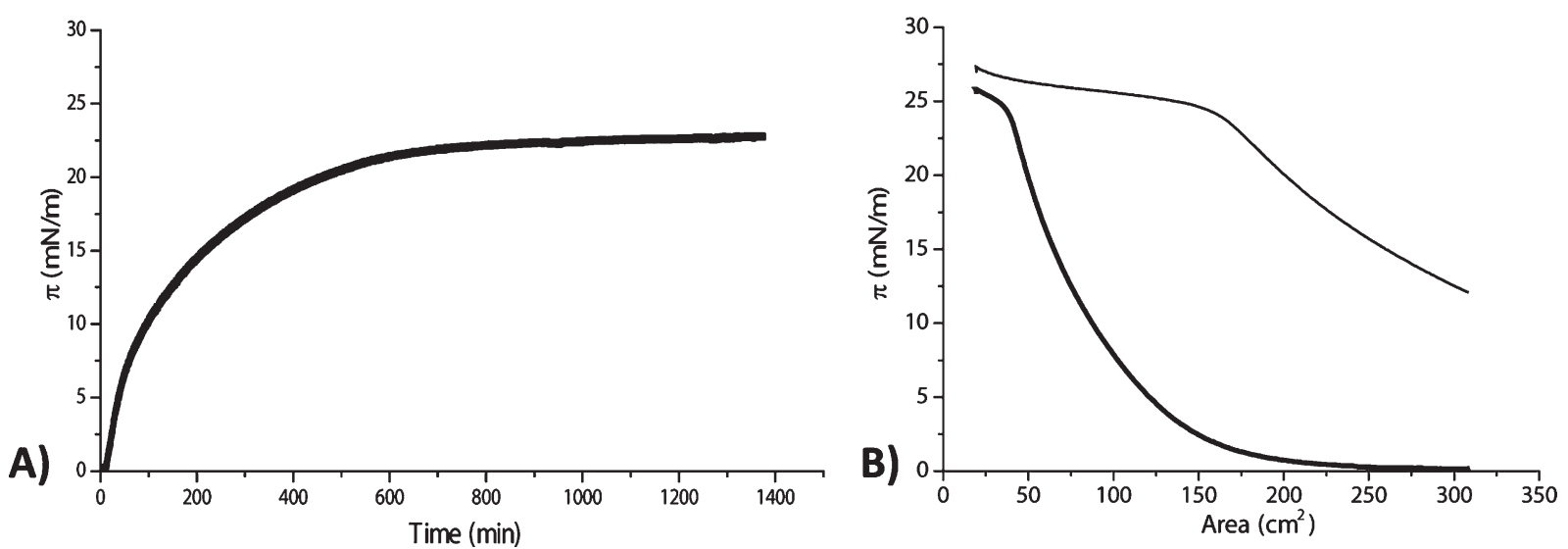

Figure 2. (A) Adsorption isotherm of $\mathrm{Fe}_{3} \mathrm{O}_{4} \mathrm{NPs}$ at the air/water interface forms an aqueous bulk solution with a concentration of $c=1.5 \times$ $10^{-3} \mathrm{mg} / \mathrm{mL}$. (B) Compression isotherms of two NP layers prepared by spreading (starting coverage: $9.7 \times 10^{-5} \mathrm{mg} / \mathrm{cm}^{2}($ thick line) and $3.9 \times 10^{-4} \mathrm{mg} / \mathrm{cm}^{2}$ (thin line)) on Milli-Q Millipore water at $20^{\circ} \mathrm{C}$.

the barrier surface, giving the water surface a convex shape in its vicinity. This might have allowed the Langmuir layer to slip underneath the barrier. To prove that this is not happening, a barrier made of POM was used. This one is quite hydrophilic (with a water contact angle of $67^{\circ}$ ), giving a concave shape to the water surface in its vicinity. In both cases, the experiments have been performed following the same procedure and the results were exactly the same, proving that there is no loss of the NP film underneath the barrier.

TEM and AFM Measurements. Transmission electron microscopy (TEM) and atomic force microscopy (AFM) techniques were used for the characterization of the transferred Langmuir layers of the $\mathrm{Fe}_{3} \mathrm{O}_{4} @ \mathrm{MEO}_{2} \mathrm{MA}_{90}$-co-OEGMA 10 NPs. TEM images were obtained using a Zeiss EM 912 Omega microscope at an acceleration voltage of $120 \mathrm{kV}$. The samples were prepared by Langmuir-Schaefer transfer of the NP interfacial layer (at surface pressures of $25 \mathrm{mN} / \mathrm{m}$ ) on a copper grid and dried under a flux of nitrogen. The AFM samples were similarly prepared, using a freshly obtained surface of mica. The measurements have been conducted in tapping mode using a Veeco (MultiMode) AFM device. The silicon AFM probes are from NanoWorld (Germany) and have the following characteristics: length, $160 \mu \mathrm{m}$; width, $45 \mu \mathrm{m}$; thickness, $4.6 \mu \mathrm{m}$; reflex Al coating on the backside; resonance frequency, $285 \mathrm{kHz}$; force constant, $42 \mathrm{~N} / \mathrm{m}$; tip radius, $10 \mathrm{~nm}$.

$\mathrm{X}$-ray Fluorescence. In situ total reflection X-ray fluorescence (TRXF) measurements at the air/water interface were performed at the beamline BW1, HASYLAB, DESY (Hamburg, Germany). The synchrotron X-ray beam was monochromated at a photon energy of $14.2 \mathrm{keV}$ by a $\mathrm{Si}$ (111) double monochromator. The beam was deflected from the horizontal plane by a gold-coated mirror. This beam touches the liquid surface at an incidence angle of $0.075^{\circ}$ that is $80 \%$ of the critical angle of total reflection for the water surface. A scintillation $(\mathrm{NaI})$ detector for the reflected $\mathrm{X}$-ray beam was used for the height adjustment of the liquid surface. The fluorescence signal was measured by the Peltier-cooled VORTEX silicon drift detector (SDD) with an entrance window placed parallel to the liquid surface. The axis of the incident beam and the view directions of the $\mathrm{NaI}$ and the SDD were lined up so that they crossed at the same point. The surface of the liquid was moved to this point before each measurement of the fluorescence signal.

$\mathrm{X}$-ray fluorescence is an element-specific technique which permits the identification of elements due to their characteristic fluorescence spectra. ${ }^{21,22}$ The grazing incidence angle of the X-ray

(21) Novikova, N. N.; Zheludeva, S. I.; Konovalov, O. V.; Kovalchuk, M. V.; Stepina, N. D.; Myagkov, I. V.; Godovsky, Y. K.; Makarova, N. N.; Y. Tereschenko, E.; Yanusova, L. G. J. Appl. Crystallogr. 2003, 36, 727.

(22) Shapovalov, V. L.; Ryskin, M. E.; Konovalov, O. V.; Hermelink, A.; Brezesinski, G J. Phys. Chem. B 2007, 111, 3927. beam limits the penetration depth to approximately $8 \mathrm{~nm}$. The fluorescence signal arises therefore from elements at the surface and is much less sensitive to contributions from the pure bulk solution.

\section{Results and Discussion}

Due to the fact that the NPs can be dispersed both in water and in chloroform, the NP films have been prepared either by adsorption of the NPs from the aqueous bulk solution (Gibbs films) or by spreading them at the interface from a chloroform solution (Langmuir films). Dispersed in water, the $\mathrm{Fe}_{3} \mathrm{O}_{4} \mathrm{NPs}$ adsorb at the air/water interface. For example, using a concentration of $1.5 \times 10^{-3} \mathrm{mg} / \mathrm{mL}$, a constant surface pressure value of approximately $23 \mathrm{mN} / \mathrm{m}$ is reached after $20 \mathrm{~h}$ (Figure 2A). Compression isotherms of Langmuir layers formed by spreading different volumes of chloroform solutions of NPs are shown in Figure 2B. By compression, a significant increase of the surface pressure is observed until to a value of approximately $25 \mathrm{mN} / \mathrm{m}$. We will call this pressure the critical pressure $\left(\pi_{\mathrm{c}}\right)$ of the NP film. During further compression, a plateau region appears at which the surface pressure increases only slightly up to a maximum value of $27 \mathrm{mN} / \mathrm{m}$.

It is worth highlighting the fact that no hysteresis of the compression-expansion isotherms is observed when the interfacial film formed by spreading is compressed to surface pressures below the critical pressure of the Langmuir layer. This suggests that no loss of the NPs from the interface occurs at surface pressures below the critical one. Using this information, we were able to calculate the interfacial mass density of the NPs which corresponds to the critical pressure and which amounts to $(7.7 \pm$ $0.6) \times 10^{-4} \mathrm{mg} / \mathrm{cm}^{2}$. This value is in good agreement with the NP value of $8.2 \times 10^{-4} \mathrm{mg} / \mathrm{cm}^{2}$, which can be calculated considering that all the NPs of the $1.5 \times 10^{-3} \mathrm{mg} / \mathrm{mL}$ aqueous solution adsorb at the air/water interface. This seems to indicate, on one hand, that all the NPs from the bulk solution adsorb at the interface and, on the other hand, that the particles spread are trapped at the interface and do not exchange with the subphase.

The surface activity of the $\mathrm{Fe}_{3} \mathrm{O}_{4} @ \mathrm{MEO}_{2} \mathrm{MA}_{90}$-co-OEGMA NPs is based on the amphiphilic character of the copolymer shell. This (oligo ethylene glycol) methyl ether methacrylate polymer has a graft structure (Figure 1) composed of an apolar carboncarbon backbone which leads to a competitive hydrophobic effect and multiple oligo(ethylene glycol) side chains of which ether oxygens form stabilizing $\mathrm{H}$-bonds with water. ${ }^{23}$ Moreover,

(23) Lutz, J.-F. Polymerization of oligo(ethylene glycol) (meth)acrylates: toward new generations of smart biocompatible materials. J. Polym. Sci., Part A: Polym. Chem. 2008, 46, 3459 . 

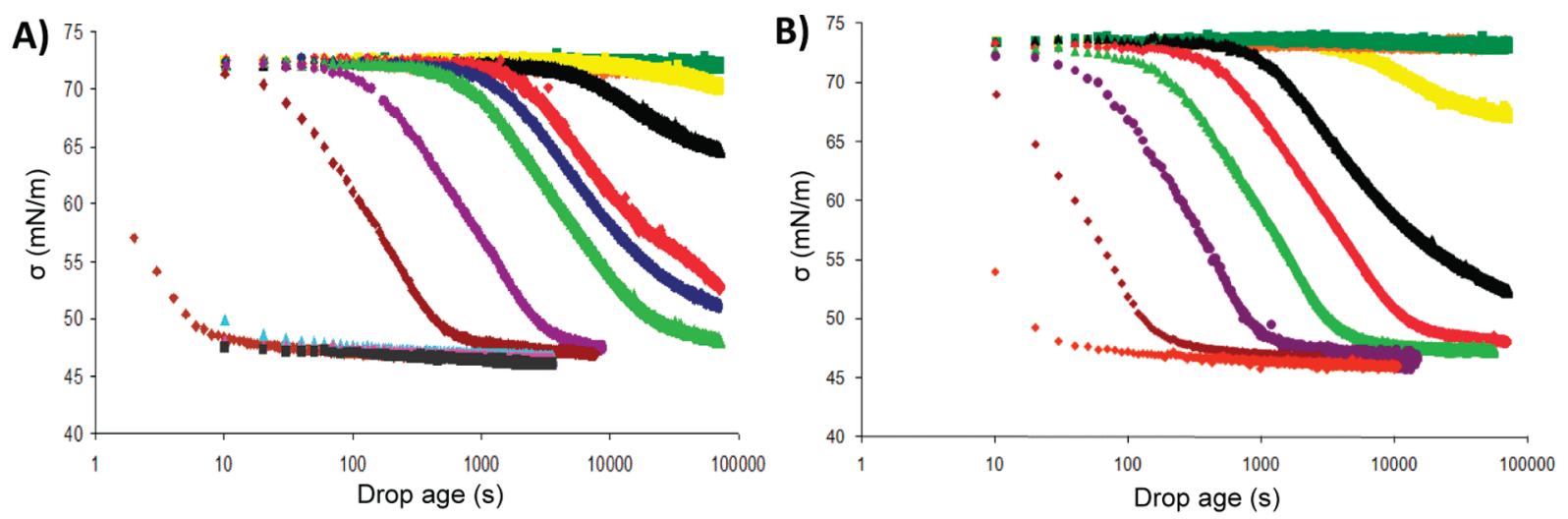

NP bulk concentration $(\mathrm{mg} / \mathrm{mL}): 5 \times 10^{-4} ; \quad 10^{-3} ; \quad 2 \times 10^{-3} ; \boldsymbol{\Delta} 3 \times 10^{-3} ; 4 \times 10^{-3} ; \bullet 4.5 \times 10^{-3}$; $\Delta 5 \times 10^{-3} ; \bullet 10^{-2} ; 2 \times 10^{-2} ; 5 \times 10^{-2} ; \Delta 10^{-1} ; \Delta 2 \times 10^{-1} ; \mathbf{\square} 4 \times 10^{-1}$.
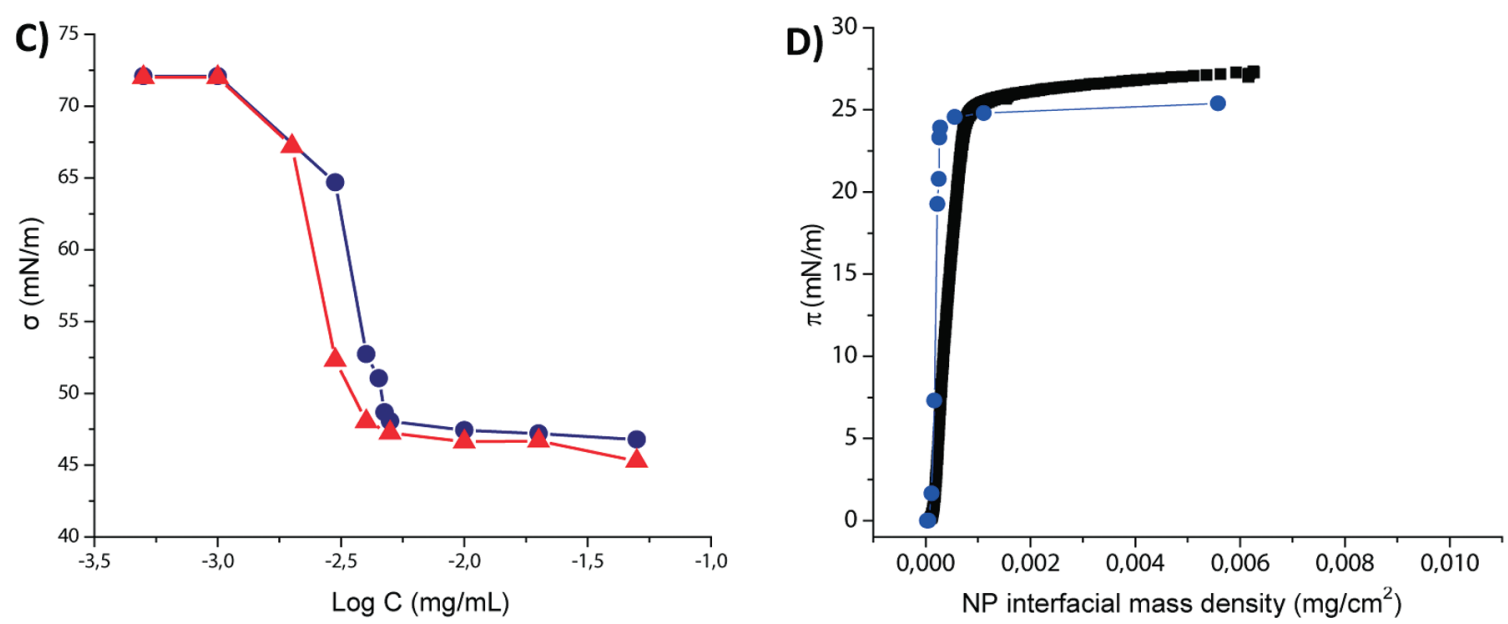

Figure 3. (A) Concentration dependence of the interfacial tension $(\sigma)$ upon adsorption of the $\mathrm{Fe}_{3} \mathrm{O}_{4} @ \mathrm{MEO}_{2} \mathrm{MA}_{90}$-co-OEGMA $\mathrm{OH}_{10} \mathrm{NPs}$ at the air/water interface. (B) Concentration dependence of the interfacial tension $(\sigma)$ upon adsorption of the pure copolymer $\mathrm{MEO}_{2} \mathrm{MA}_{90}$-coOEGMA $_{10}$ at the air/water interface. (C) Variation of the interfacial tension $(\sigma)$ with the logarithm of the bulk concentration of the NPs (blue circles) and of the copolymer (red triangles). (D) Variation of the surface pressure with the interfacial mass density of the NPs (blue circles, PAT data; black line, data from the compression isotherms-Figure 2B).

the ethylene oxide motif can adopt a configuration with the oxygen atoms on one side of the molecule and with the two methylene groups on the other one, thus giving the molecule both a hydrophilic and a hydrophobic surface. ${ }^{24}$ Without going into more detail, it is worth mentioning that, in this special case, the copolymer hydrophobicity can be tuned by changing the molar fraction of the 2 monomers, $\mathrm{MEO}_{2} \mathrm{MA}$ and OEGMA. ${ }^{23}$ The copolymer becomes more hydrophobic by increasing the molar fraction of $\mathrm{MEO}_{2} \mathrm{MA}$.

A more detailed study of the concentration-dependent adsorption of the $\mathrm{Fe}_{3} \mathrm{O}_{4} @ \mathrm{MEO}_{2} \mathrm{MA}_{90}$-co-OEGMA 10 NPs from aqueous solutions at the air/water interface was performed using the profile analysis tensiometer (PAT).

Figure $3 \mathrm{~A}$ shows a series of pendant drop tensiometer measurements for different concentrations of $\mathrm{Fe}_{3} \mathrm{O}_{4} @ \mathrm{MEO}_{2} \mathrm{MA}_{90^{-}}$

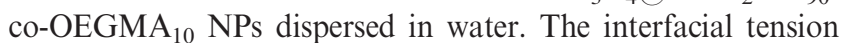
decreases with time and approaches an equilibrium value, which decreases with increasing concentration of the NPs. For a NP concentration of $3 \times 10^{-3} \mathrm{mg} / \mathrm{mL}$, a minimum surface tension of $65 \mathrm{mN} / \mathrm{m}$ was reached after $20 \mathrm{~h}$. For more concentrated colloidal solutions (concentrations above $10^{-2} \mathrm{mg} / \mathrm{mL}$ ), the surface tension $(\sigma)$ of water was reduced from $72 \mathrm{mN} / \mathrm{m}$ to an equilibrium value of $47 \mathrm{mN} / \mathrm{m}$ in less than $2 \mathrm{~h}$. The colloidal suspensions of NPs in a concentration range of $10^{-2}$ to $4 \times 10^{-1} \mathrm{mg} / \mathrm{mL}$ gave very similar

(24) Shuler, R. L.; Zisman, W. A. J. Phys. Chem. 1970, 74, (7). values of the equilibrium interfacial tension, suggesting that the maximum coverage of the interface with nanoparticles was reached. The different slopes of the $\sigma=f$ (time) curves highlight the different stages of adsorption of the NPs to the interface. In the first stage, the diffusion-limited transport of the NPs close to the interface determines the kinetics. This process is strongly concentration dependent (see Figure 3A). The rapid decrease of the surface tension characterizes the second stage, which describes the adsorption to the surface. In the last stage, the surface tension decrease slows down, reaching a constant value. In this stage, a rearrangement of the NPs at the interface seems to be the limiting process.

Moreover, Figure 3B shows the experimentally obtained curves of the surface tension versus time for the pure copolymer $\mathrm{MEO}_{2} \mathrm{MA}_{90}$-co-OEGMA 10 . We note that, at the same bulk concentration $(\mathrm{mg} / \mathrm{mL})$, the copolymer adsorption is much faster than that of the NPs, and the final equilibrium surface tension is lower. However, the equilibrium value of the surface tension is exactly the same at high enough concentrations for both systems. The comparison with the results obtained using the Langmuir balance shows that the final plateau obtained at an interfacial tension of $47 \mathrm{mN} / \mathrm{m}$ (Figure $3 \mathrm{~B}$ ) corresponds well with the critical pressure of the Langmuir monolayer. This shows that both the NPs as well as the pure copolymer attained a maximum coverage (highest possible packing density) of the interface. 

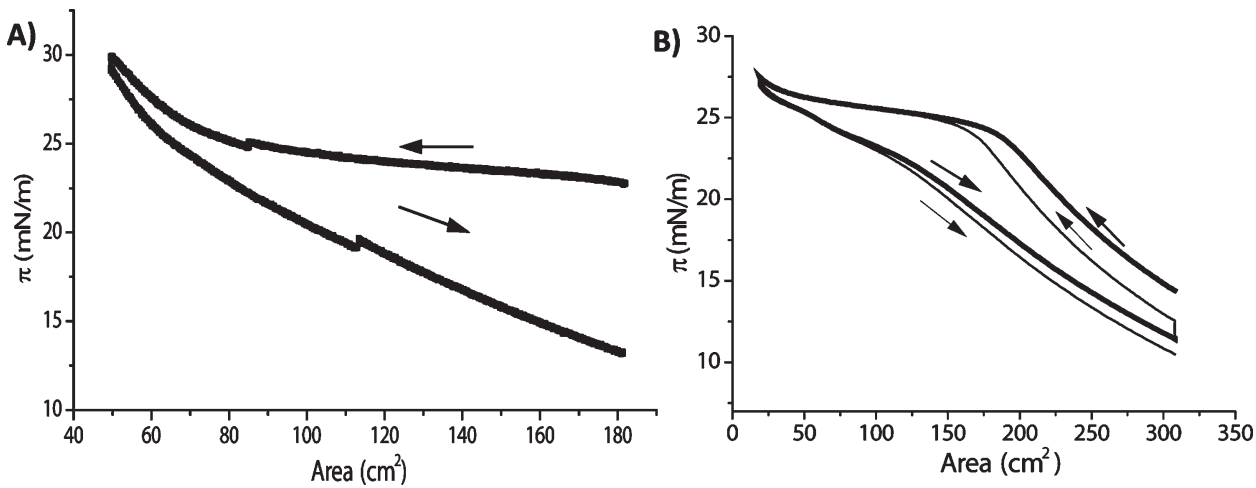

Figure 4. (A) Compression-expansion isotherm of the NP layer after the adsorption from an aqueous bulk solution $\left(c=1.5 \times 10^{-3} \mathrm{mg} / \mathrm{mL}\right)$. (B) Two compression-expansion isotherms (1st cycle, thick line; 2nd cycle, thin line) of a layer of NPs prepared by spreading (starting coverage $3.9 \times 10^{-4} \mathrm{mg} / \mathrm{cm}^{2}$ ) on Milli-Q Millipore water at $20^{\circ} \mathrm{C}$.

Furthermore, Figure 3C shows the equilibrium surface tension values obtained from the adsorption of the NPs at the air/water interface as a function of the bulk NP concentration. The profile of the curve shows a continuous decrease of the surface tension with increasing concentration of the NPs up to a "critical" concentration of around $10^{-2} \mathrm{mg} / \mathrm{mL}$ (Figure 3C). Translating this concentration into a surface coverage, considering that all NPs migrate from bulk to the interface and that the experimental drop has a constant volume of $15 \mathrm{~mm}^{3}$ and a drop surface of $26.85 \mathrm{~mm}^{2}$, one obtains a surface mass density of $5.6 \times 10^{-4} \mathrm{mg} / \mathrm{cm}^{2}$. Additionally, Figure 3D reveals the same profile of the surface pressure against the surface coverage of the interface with NPs independent of the way the surface coverage is obtained (either by spreading or by adsorption from the bulk solution). The two sets of data are in good agreement until the critical surface pressure is reached. Therefore, two major observations need to be highlighted. First, for the NP films formed by spreading, the NPs are trapped at the air/water interface and can be compressed until a critical value of the surface pressure is reached. Second, all NPs from the bulk adsorb to the interface until reaching a maximum coverage of the interface, which corresponds to values between $6 \times 10^{-4}$ to $8 \times 10^{-4} \mathrm{mg} / \mathrm{cm}^{2}$ and a critical equilibrium surface pressure of $25 \mathrm{mN} / \mathrm{m}$.

For low concentrations, the amount of NPs is evidently not enough to reach a complete coverage of the interface. In this case, the adsorption of the NPs to the interface is the favored process. At higher bulk concentrations, the critical packing density of the adsorption layer is reached, and the remaining NPs have to stay in the aqueous bulk phase.

The acquired data allowed us to calculate (see Supporting Information) that the $\mathrm{Fe}_{3} \mathrm{O}_{4}$ cores are at the interface at $26-33 \mathrm{wt}$ $\%$. These values are in good agreement with the results obtained from the TGA. Furthermore, at the same copolymer concentration, the adsorption kinetics and the final minimum surface tensions are very similar for the two systems (copolymer-capped NPs and pure copolymer). This indicates that the adsorption process is determined by the copolymer, the influence of the NPs being negligible. This behavior is different from that of classical water-soluble surfactants for which the equilibrium between the interfacial and the bulk concentration is reached. Moreover, at concentrations above the critical one, the NP concentration at the interface is constant.

The system under study is distinguished by the fact that it forms stable Langmuir layers as well as Gibbs layers. From this comparison, we can derive a more quantitative understanding.

The compression-expansion isotherms recorded for the adsorbed NP layers and for the NP Langmuir layers formed by spreading show very similar behavior (Figure 4A,B). During compression and expansion of the NP layer, a pronounced hysteresis was observed. Figure 4B shows the $\pi / A$ isotherms upon repeated compression and expansion cycles. The two compression and expansion curves, respectively, have similar profiles. Using three compression-expansion cycles, the compression isotherms shift each time to smaller area values and shorter plateaus.

It is also important to highlight the fact that the critical surface pressure characterizing the plateau region agrees well with the equilibrium adsorption surface pressure observed for high enough bulk concentrations.

The characteristics of the $\pi / A$ isotherms, especially the existence of a plateau at a value of the surface pressure similar to that of the adsorption equilibrium and the hysteresis observed upon compression-expansion of the layer, indicate that at surface pressures above the critical pressure either the NPs form an irreversible multilayer film on top of the water surface, which does not respread after expansion, or they are squeezed out from the interface and redispersed into the aqueous bulk.

In order to distinguish between the two possible scenarios, the NP Langmuir film was further investigated in situ using the X-ray fluorescence technique, and also after the transfer onto a solid support by using transmission electron microscopy (TEM) and atomic force microscopy (AFM).

The X-ray fluorescence experiments were performed to prove the presence of the $\mathrm{Fe}_{3} \mathrm{O}_{4} \mathrm{NPs}$ at the air/water interface. The $\mathrm{X}$-ray fluorescence $\mathrm{Fe} \mathrm{K} \alpha(6.4 \mathrm{keV})$ and $\mathrm{Fe} \mathrm{K} \beta(7.06 \mathrm{keV})$ lines, obtained for a NP film compressed to $25 \mathrm{mN} / \mathrm{m}$ (in the plateau region, the $\mathrm{X}$ mark in Figure $5 \mathrm{~A}$ ), are shown in Figure $5 \mathrm{~B}$. The presence of $\mathrm{Fe}$, and therefore of the $\mathrm{Fe}_{3} \mathrm{O}_{4} \mathrm{NPs}$, at the air/water interface is confirmed by the characteristic peaks. Moreover, the AFM and TEM images presented in Figure 5C,D confirm the presence of the $\mathrm{Fe}_{3} \mathrm{O}_{4} @ \mathrm{MEO}_{2} \mathrm{MA}_{90}$-co-OEGMA 10 NPs at the interface. The discrepancy of the TEM and AFM pictures might arise from a deficient transfer of the NP layer onto the different solid supports or could be due to an inhomogeneous distribution of the NPs at the interface. However, the TEM and AFM measurements indicate clearly that these NPs form monolayers at the air/water interface but not multilayers.

In order to prove that upon compression to surface pressures above the critical pressure the NPs are squeezed out from the interface into the aqueous subphase, the following experiment was performed. A layer of NPs was formed only on one side of a one-barrier Langmuir trough (Figure 6A) by spreading an NP dispersion in chloroform at the air/water interface. By using two surface pressure microbalances $\left(\mathrm{P}_{1}\right.$ and $\left.\mathrm{P}_{2}\right)$, we measured simultaneously the surface pressure on both sides of the barrier. After the NP film was formed and compressed to a minimal surface 


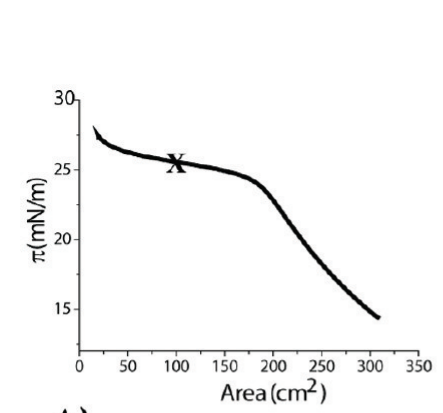

A)

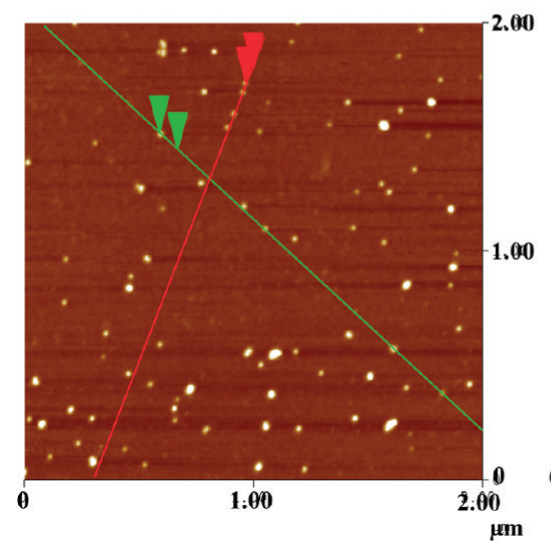

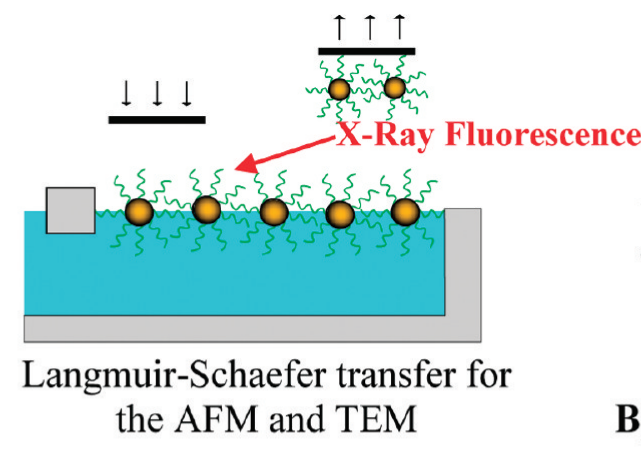

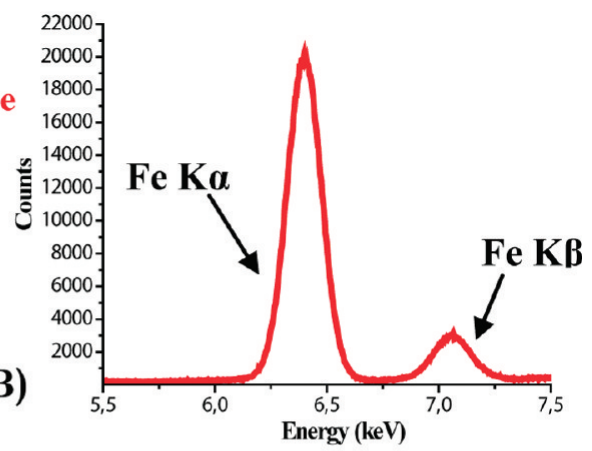

D)

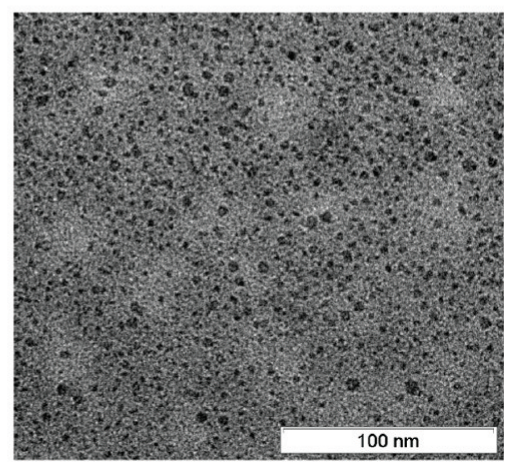

Figure 5. (A) Schematic representation of the NP layer at the air/water interface. The $\mathrm{X}$ mark on the compression isotherm (at $25 \mathrm{mN} / \mathrm{m}$ ) shows the state where the film was investigated by TRXF, AFM, and TEM. (B) X-ray fluorescence showing the Fe K $\alpha$ and Fe K $\beta$ peaks. (C) AFM image and height profile showing that mainly the NP core can be measured. (D) TEM image of the NP Langmuir layer transferred on mica or on a copper grid, respectively, by the Langmuir-Schaefer technique.

area, the barrier was stopped in this position for more than one hour and the surface pressure changes have been recorded in time on both sides of the barrier. No variation of the surface pressure was recorded during this time on either side. On the spreading compartment, a constant value of $28 \mathrm{mN} / \mathrm{m}$ was measured, and a value of $0 \mathrm{mN} / \mathrm{m}$ on the other side of the barrier. However, after expansion of the initial NP Langmuir layer (compression toward the surface pressure microbalance $\mathrm{P}_{2}$, Figure $6 \mathrm{~B}$ ) a decrease of the surface pressure on the spreading area and an increase in the surface pressure on the right side was measured (Figure 6C,D). After spreading new NPs twice and repeating the above-described experiment, an increase of the surface pressure up to a value of $23 \mathrm{mN} / \mathrm{m}$ could be recorded by the surface pressure microbalance $\mathrm{P}_{2}$ (Figure 6D).

The drastically reduced surface pressure recorded after the expansion of the NP layer (Figure 6C, red line) suggests that the compression of the NP layer to pressures above the critical pressure leads to the desorption of the NPs from the interface into the subphase. Additionally, the desorbed NPs adsorb slowly at the free water surface on the right compartment, which offers a large unoccupied surface area.

A more detailed analysis of the compression-expansion isotherm from Figure 6C (1st cycle, red line) reveals that the decrease in the surface pressure from $19.6 \mathrm{mN} / \mathrm{m}$ (corresponding to an interfacial NP concentration of $5.92 \times 10^{-4} \mathrm{mg} / \mathrm{cm}^{2}$ ) to $1.6 \mathrm{mN} / \mathrm{m}$ (corresponding to an interfacial NP concentration of $1.79 \times$ $10^{-4} \mathrm{mg} / \mathrm{cm}^{2}$ ) is due to the desorption of $1.18 \times 10^{-1} \mathrm{mg} \mathrm{Fe}_{3} \mathrm{O}_{4}$ NPs from the interface. Once in the $300 \mathrm{~mL}$ aqueous subphase, the NPs will generate a bulk concentration of $3.95 \times 10^{-4} \mathrm{mg} / \mathrm{mL}$. The surface pressure on the right side of the barrier remained zero but increased $8.3 \mathrm{mN} / \mathrm{m}$ on compression (Figure 6D, first compression), corresponding to a surface coverage of $2.71 \times 10^{-4}$ $\mathrm{mg} / \mathrm{cm}^{2}$ and a mass of $8.65 \times 10^{-3} \mathrm{mg} \mathrm{Fe}_{3} \mathrm{O}_{4} \mathrm{NPs}$. Taking the different surface area/volume ratio into account, the obtained data are in good agreement with the PAT results. Additionally, these data prove that, at this low bulk concentration $\left(3.95 \times 10^{-4} \mathrm{mg} / \mathrm{mL}\right)$ and in only one hour, $7 \%$ of the NPs are adsorbed to the interface, with the rest of $0.11 \mathrm{mg}$ still dispersed in the subphase. All these values prove that the data acquired by compression of the Langmuir films are in perfect agreement with the profile analysis tensiometer results.

Moreover, the fact that the NPs are trapped at the air/water interface for surface pressures below the critical surface pressure of the layer was confirmed by the use of the two surface pressure microbalance Langmuir trough. This was expressed by the lack of increase of the surface pressure recorded on the right side of the barrier $(0 \mathrm{mN} / \mathrm{m})$ after compression of the spread film to only $22 \mathrm{mN} / \mathrm{m}$, an equilibration time of $80 \mathrm{~min}$, followed by the expansion of the layer. This sustains the lack of hysteresis recorded for the compression-expansion isotherms performed below the critical pressure of the Langmuir layer, as mentioned above.

All these data suggest that the redistribution of the NPs into the subphase occurs only above the critical pressure. This behavior can be understood by the hydrophobicity of the surrounding copolymer. Sauer et al. explained the collapse pressure of a layer of polyethylene oxide (PEO) by segmental desorption into the aqueous phase. ${ }^{25}$ The desorption behavior of the polyethylene oxide Langmuir layers was discussed and proven by some other studies as well. ${ }^{17,26}$ Moreover, in our case, a detailed study (manuscript in preparation) indicates that the NP interfacial behavior is dictated by the conformational change of the copolymer chains

(25) Sauer, B. B.; Kawaguchi, M.; Yu, H. Macromolecules 1987, 20, 2732-2739.

(26) Kuzmenka, D. J.; Granick, S. Macromolecules 1988, 21, 779. 

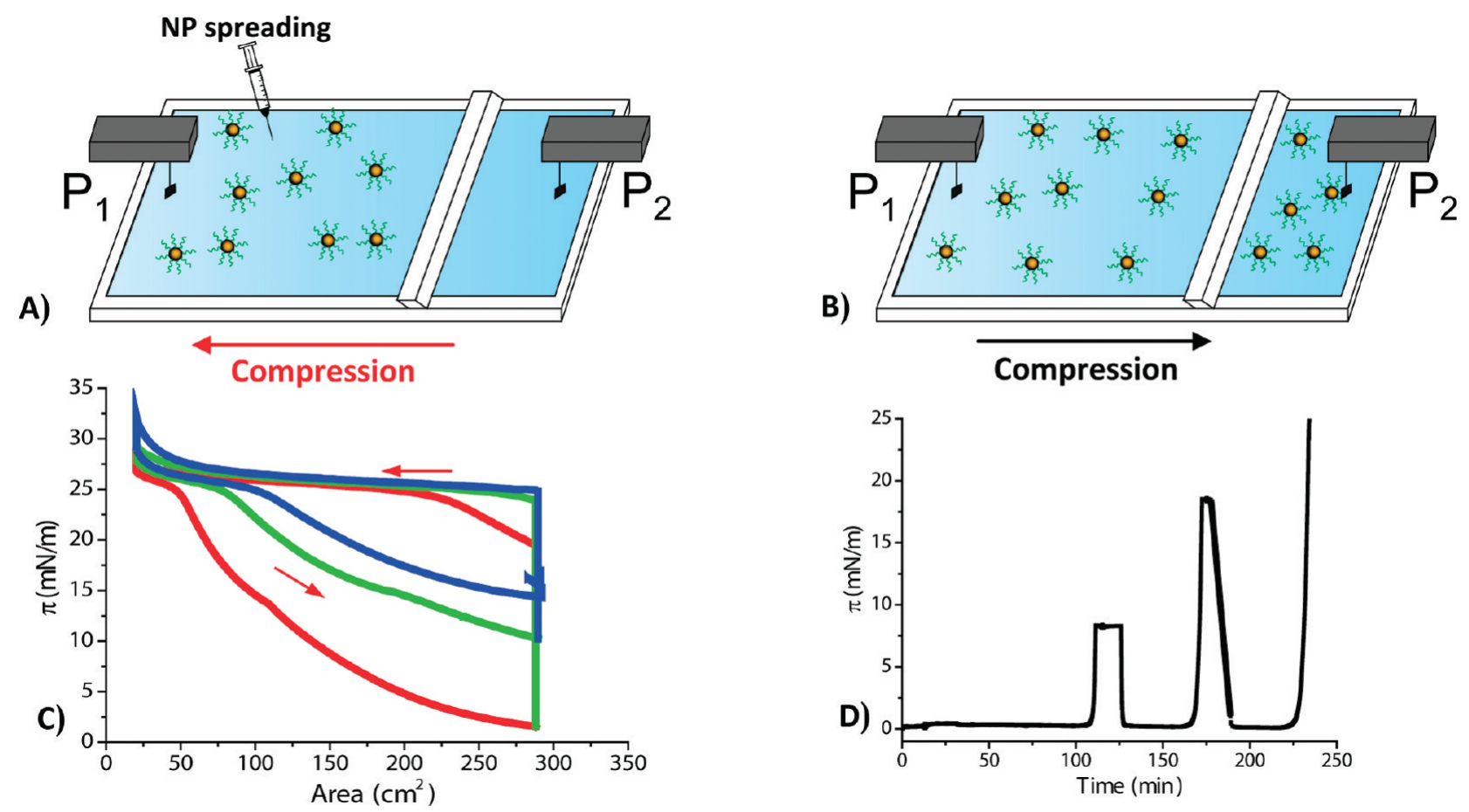

Figure 6. (A,B) Schematic representations of the setup, the procedure used and the experimental observations. (C) Three compression-expansion isotherms of the NP layers (1st cycle, red line; 2nd cycle, green line; 3rd cycle, blue line) obtained on the spreading area. (D) Variation of the surface pressure in time (measured by the surface pressure microbalance $\mathrm{P}_{2}$ ) on the right side of the barrier. Note that the right compartment was compressed after $100 \mathrm{~min}$. The corresponding compression-expansion cycles of the right compartment are shown in $\mathrm{D}$ as well.

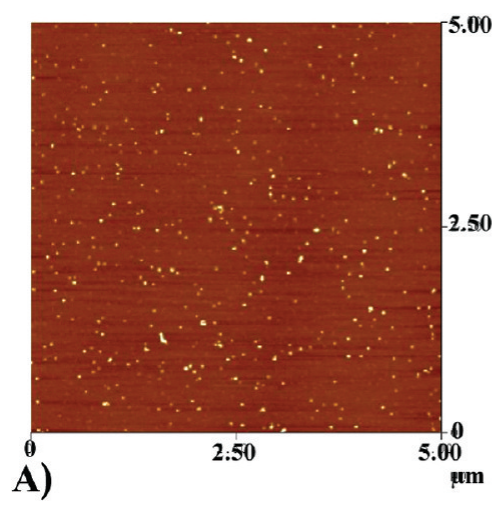

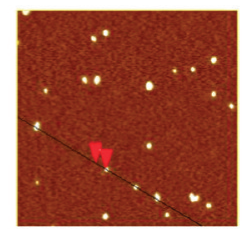

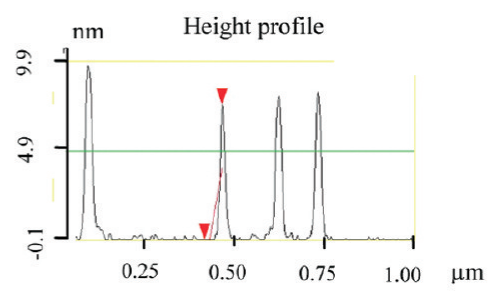

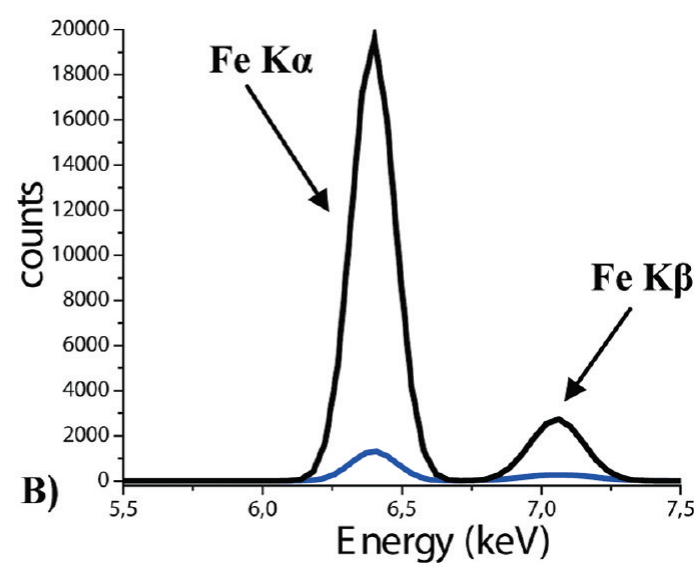

Figure 7. (A) AFM image of the NP Langmuir layer $(23.5 \mathrm{mN} / \mathrm{m})$ transferred onto mica support by the LS technique and the height profile measured along the line (detail image $1 \times 1 \mu \mathrm{m}^{2}$ ). (B) X-ray fluorescence spectrum in the region of the Fe K $\alpha$ and Fe K $\beta$ peaks of the NP interfacial layer at $1.1 \mathrm{mN} / \mathrm{m}$ (blue line) and $5.4 \mathrm{mN} / \mathrm{m}$ (black line).

from the brush-like structure in the solution to a pancake conformation at the air/water interface.

It seems that the NPs, after spreading from a chloroform solution, are in a metastable state, being trapped at the air/water interface. The film is even stable in contact with a NP free subphase. Compression of such a layer leads only to their approach and rearrangement. Only above the critical pressure do the NPs desorb into the aqueous phase. Once in the aqueous subphase, due to their hydrophobicity, the NPs will again adsorb at the free air/water interface, namely, on the right side of the barrier (Figure 6B,D), and not on the left side, where a high packing density is already reached and indicated by the high surface pressure of $28 \mathrm{mN} / \mathrm{m}$. It remains unclear whether the equilibrium on the plateau is a dynamic equilibrium (exchange of NPs between the bulk and the interface) or not.

The presence of NPs on the right side of the barrier was further confirmed by AFM measurements of samples prepared by the Langmuir-Schaefer technique. A representative AFM image obtained for the NP layer transferred at a surface pressure of $23.5 \mathrm{mN} / \mathrm{m}$ onto freshly prepared mica is shown in Figure 7A. These data are additional proofs of the presence of the adsorbed $\mathrm{Fe}_{3} \mathrm{O}_{4} @ \mathrm{MEO}_{2} \mathrm{MA}_{90}$-co-OEGMA 10 NPs at the interface (on the right side of the barrier). The same lack of dense packing of the metallic cores is observed as discussed above. Additionally, the enrichment of the right compartment with the iron oxide NPs was determined by measuring in situ X-ray fluorescence spectra. 
An increase of the intensity of the characteristic $\mathrm{Fe} \mathrm{K} \alpha$ and $\mathrm{Fe} \mathrm{K} \beta$ $\mathrm{X}$-ray fluorescence peaks upon compression of the adsorption layer was recorded (Figure 7B). This increase of the fluorescence intensity which does not correlate in a linear manner with the compression ratio could be an additional indication of the nonhomogeneous distribution of the NPs, as observed in the AFM images.

\section{Conclusions}

The air/water interfacial properties of $\mathrm{Fe}_{3} \mathrm{O}_{4} @ \mathrm{MEO}_{2} \mathrm{MA}_{90^{-}}$ co-OEGMA ${ }_{10}$ NPs, previously described as very promising nanotools for biomedical applications, were the topic of this study.

Due to the inherent hydrophobic-hydrophilic properties of the copolymer and despite their high solubility in water, the NPs proved to be surface active. Their surface activity is concentration- and time-dependent. However, these NPs do not behave as classical amphiphiles. Therefore, the Gibbs layer is not a Gibbs layer in the traditional sense. Once adsorbed at the air/water interface, they do not exchange with NPs in bulk, but they are trapped at this interface. This means that all NPs from the bulk adsorb to the interface until reaching maximum coverage of the interface, which corresponds to values between $6 \times 10^{-4}$ and $8 \times$ $10^{-4} \mathrm{mg} / \mathrm{cm}^{2}$ and a critical equilibrium surface tension of $47 \mathrm{mN} / \mathrm{m}$. For low bulk concentrations, the amount of NPs is simply not sufficient for a complete coverage of the interface. At higher bulk concentrations, the critical packing density of the adsorption layer is reached and the remaining NPs stay in the aqueous bulk phase without being exchanged with adsorbed NPs.

The interfacial films of the $\mathrm{Fe}_{3} \mathrm{O}_{4} @ \mathrm{MEO}_{2} \mathrm{MA}_{90}$-co-OEGMA 10 NPs, once formed by adsorption or by spreading on the water surface, are extremely stable up to the critical surface pressure of approximately $25 \mathrm{mN} / \mathrm{m}$. Further compression of the film leads to a pronounced plateau above the critical pressure. A large hysteresis is obtained upon compression-expansion of the NP layer. By using an original setup with a one barrier Langmuir trough equipped with two surface pressure microbalances placed on both sides of the moving barrier, we have shown that the NPs are squeezed out from the interface by compression of the film above the critical packing density, and redispersed into the aqueous bulk (Figure 8).

The readsorption of the squeezed-out NPs on the other side of the barrier was proved by surface pressure measurements, in situ X-ray fluorescence, as well as TEM and AFM experiments of the transferred layers. In this work, we could use the Langmuir monolayer to obtain an exact relationship between weight concentration on a surface for the copolymer as well as for the copolymer-

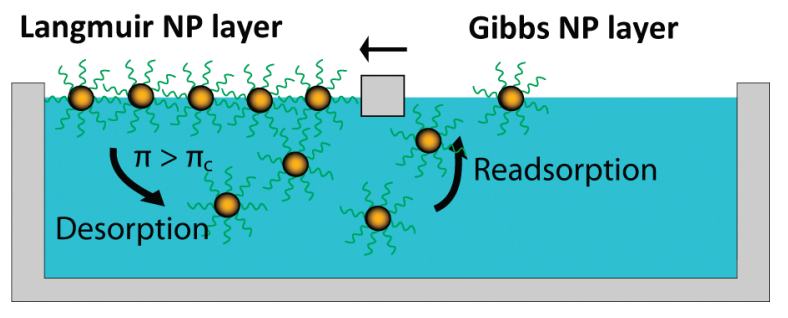

Figure 8. Schematic representation of the desorption/readsorption of the $\mathrm{Fe}_{3} \mathrm{O}_{4} @ \mathrm{MEO}_{2} \mathrm{MA}_{90}$-co-OEGMA 10 NPs from/at the air/ water interface.

NP films and from this derive data on interfacial weight losses and dynamics of adsorption.

To conclude, we showed that the $\mathrm{Fe}_{3} \mathrm{O}_{4} @ \mathrm{MEO}_{2} \mathrm{MA}_{90^{-}}$ co-OEGMA ${ }_{10}$ NPs are hydrophobic enough for the adsorption at the air/water interface, giving an equilibrium surface pressure of $25 \mathrm{mN} / \mathrm{m}$ and formation of stable films up to this value. Once trapped at the air/water interface, the thermodynamically metastable NP films are energetically stabilized and kinetically longterm stable. Furthermore, we proved that the NPs are hydrophilic enough to be squeezed out from the interface if the packing density exceeds a critical value and to be dispersed in the aqueous subphase. Therefore, we revealed the ability of the $\mathrm{Fe}_{3} \mathrm{O}_{4} @$, $\mathrm{MEO}_{2} \mathrm{MA}_{90}$-co-OEGMA ${ }_{10}$ NPs to interchange the hydrophilic/ hydrophobic character. This is a very important aspect, considering our interest in studying and understanding the ability of these NPs to cross biological membranes. The studies of the interaction of these stimuli-responsive NPs with model membranes, as well as their response to temperature changes and different ion strength media, are still ongoing and will be reported in the near future.

Acknowledgment. We thank Dr. Reinhard Miller for giving us the possibility to perform the dynamic surface tension measurements in his group, Dr. Vincent Pradines for help and scientific discussions, Anneliese Heilig for the AFM measurements, Rona Pitschke for the TEM measurements, and Mandy Meckelburg for the preparation of the NPs colloidal dispersions. We thank HASYLAB at DESY, Hamburg, Germany, for beamtime and excellent support. This work was supported by the Max Planck Society.

Supporting Information Available: Additional data and figures. This material is available free of charge via the Internet at http://pubs.acs.org. 